\title{
The utility of measuring the apparent diffusion coefficient for peritumoral zone in assessing infiltration depth of endometrial cancer
}

Lei Deng $^{1 \dagger}$, Qiu-ping Wang ${ }^{1 \dagger}$, Rui Yan ${ }^{2}$, Xiao-yi Duan ${ }^{3}$, Lu Bai ${ }^{1}$, Nan Yu ${ }^{4}$, You-min Guo ${ }^{1}$ and Quan-xin Yang ${ }^{1 *}$

\begin{abstract}
Background: The invasion depth of endometrial cancer is one of the most important prognosis factors. The aim of the current study was to investigate the diagnostic value of the apparent diffusion coefficient (ADC) of the peritumoral zone for assessing the infiltration depth of endometrial cancer.

Methods: An institutional review board approved this prospective study, and all study participants provided informed consent. A total of 58 patients (mean age $54 \pm 8.3$ years, range 34-69 years) with endometrial cancer were prospectively enrolled. Two radiologists assessed all preoperative magnetic resonance images with $\mathrm{T} 1$, $\mathrm{T} 2$, and diffusion-weighted imaging, and determined the location of the deepest invasion of the tumor. The peritumoral zone was defined as a 5-mm-thick zone surrounding and adjacent to the cancerous endometrium. The mean ADC $(A D C m)$ values of the tumor and the peritumoral zone were measured. Sensitivity, specificity, positive and negative predictive values, and the area under the receiver operating characteristic curve (Az) were calculated for visual inspection, and an ADC cutoff value for the peri-endometrial zone was determined for predicting the myometrial invasion depth.
\end{abstract}

Results: The ADCm values of tumors and peritumoral zones were $0.83 \times 10^{-3} \mathrm{~mm}^{2} / \mathrm{sec}$ and $1.06 \times 10^{-3} \mathrm{~mm}^{2} / \mathrm{sec}$, respectively. There was no significant difference between the $A D C m$ values of the tumors in the superficial and deep myometrial invasion groups $(P>0.05)$. However, the $A D C m$ value at the peritumoral zone in the deep myometrial invasion group $\left(1.23 \times 10^{-3} \mathrm{~mm}^{2} / \mathrm{sec}\right)$ significantly differed from that in the superficial myometrial invasion group $\left(0.99 \times 10^{-3} \mathrm{~mm}^{2} / \mathrm{sec}\right)(p=0.005)$. In assessments of deep myometrial invasion, the sensitivity, specificity, negative predictive value, and positive predictive value were $0.58,0.93,0.84$, and 0.77 , respectively, for the ADCm cutoff value of the peritumoral zone, and $0.71,0.80,0.87$, and 0.60 . respectively, for visual inspection. The accuracy of myometrial invasion depth assessment using the ADCm cutoff value and visual inspection were 83 and $78 \%$, respectively. The Az for both was 0.76 .

Conclusion: $\mathrm{ADCm}$ at the peritumoral zone can predict deep myometrial invasion of endometrial cancer. This value can therefore enhance confidence in preoperative endometrial cancer evaluation, and when tailoring surgical approaches.

Keywords: Diagnostic imaging, Diffusion, Endometrial carcinoma, Endometrial neoplasm, Magnetic resonance imaging

\footnotetext{
* Correspondence: quanxy6285@163.com

${ }^{\dagger}$ Lei Deng and Qiu-ping Wang contributed equally to this work.

${ }^{1}$ Department of Radiology, the First Affiliated Hospital, Xi'an Jiaotong

University Xi'an, \#277, Yanta West Road, Xi'an 710061, Shaanxi, China

Full list of author information is available at the end of the article
}

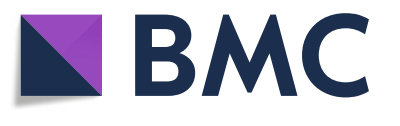

(c) The Author(s). 2018 Open Access This article is distributed under the terms of the Creative Commons Attribution 4.0 International License (http://creativecommons.org/licenses/by/4.0/), which permits unrestricted use, distribution, and

reproduction in any medium, provided you give appropriate credit to the original author(s) and the source, provide a link to the Creative Commons license, and indicate if changes were made. The Creative Commons Public Domain Dedication waiver (http://creativecommons.org/publicdomain/zero/1.0/) applies to the data made available in this article, unless otherwise stated. 


\section{Background}

Endometrial cancer is the sixth most common malignant disorder in women worldwide [1]. Its prognosis depends on multiple factors,with the depth of myometrial invasion being one of most important [2]. This depth may be used as a surrogate marker to determine possible lymphovascular space invasion and the risk of lymph node metastases [3, 4]. The prevalence of lymph node metastases increases from $3 \%$ with superficial myometrial invasion to $46 \%$ with deep myometrial invasion [5], and the recurrence risk was reportedly intermediate to moderately high in patients with deep myometrial invasion [6]. Therefore, accurate preoperative delineation of the myometrial invasiveness of endometrial cancer is essential.

Magnetic resonance imaging (MRI) is recommended for the management and preoperative staging of endometrial cancer [7]. Recently, diffusion-weighted (DW) imaging has been introduced to better evaluate tissue composition in gynecologic tumors [8]. DW images can qualitatively analyze the myometrial invasion depth of endometrial cancer $[9,10]$, especially in combination with T2WI [11]. Apparent diffusion coefficient (ADC) values allow normal endometrium or benign lesions to be differentiated from endometrial carcinoma [12, 13]; however, they could not quantitatively diagnose the myometrial infiltration depth of endometrial cancer [13, 14]. According to FIGO 2009, a tumor that invades $\geq 50 \%$ of the myometrium is defined as deep myometrium infiltration of endometrial cancer [15], whereas superficial myometrial invasion is defined as tumor invasion $<50 \%$ of the myometrium invasion depth. The integration of the junctional zone is very important for the assessment of myometrium infiltration depth [16]. However, in the presence of pitfalls such as a loss of junctional zone definition, poor tumor to myometrium contrast, myometrial compression by polypoid tumor, leiomyomas, and adenomyosis, morphologic inspection are challenging for the accurate assessment of myometrial invasion depth [17]. As the previously studies reported, in a normal uterus, the ADC value of junctional zone was the lowest among the three layers and the highest in the outer myometrium $[18,19]$. The most important prognostic factor is the variation of invasion depth with different degrees of integration of the junctional zone, as mentioned above. Hence, a change in diffusion may also be present in the peritumoral zone of endometrial cancer.

The purpose of this study was to explore the diagnostic value of the ADC value of the peritumoral zone for predicting the myometrial invasion depth of endometrial cancer in comparison with the ADC value of the tumor.

\section{Methods}

\section{Study population}

Our institutional review board approved this prospective study, and all study participants provided informed consent.
All patients were histopathologically confirmed to have primary untreated endometrial cancer via fractional dilatation and curettage or biopsy. Patients were excluded if they had (a) any contraindications for MRI (such as a cardiac pacemaker or defibrillator, insulin pump, aneurysm clip, implanted neural stimulator, cochlear implant, or metal shrapnel or bullet); (b) pelvic or hip metal prostheses; (c) not provided informed consent; (d) any contraindications for surgery; or (e) unavailable postoperative histological reports.

Between April 2012 and January 2014, based on surgery and pathology, a total of 58 consecutive patients (mean age $54 \pm 8.3$ years, range 34-69 years) were enrolled. All the patients underwent pelvic MRI as part of their initial staging before surgery.

\section{Imaging protocol}

The MRI was performed with a 3.0-T MRI unit (Signa HDx 3.0 T, GE Medical Systems, GE Healthcare, Waukesha, Wis, USA) with an 8US TORSOPA coil. All subjects had fasted for $6 \mathrm{~h}$ and were trained to hold their breaths at the end of expiration before scanning. For all examinations, patients were placed in the supine position and had a partially filled bladder. T1-weighted, T2-weighted, and DW images of the pelvis were acquired. Fast spin-echo T2-weighted images were initially obtained in the sagittal, axial, and coronal planes with the following parameters: repetition time (TR)/echo time (TE) 3680-6240 ms/85$89 \mathrm{~ms}$, field of view $30-35 \mathrm{~cm}$, number of acquired signals 2, section thickness $5 \mathrm{~mm}$, and bandwidth 35.71-83.33 $\mathrm{KHz}$. Following this sequence, axial fast spin-echo T1-weighted images were acquired with the following parameters: number of acquired signals 2, section thickness $5 \mathrm{~mm}$, and bandwidth $50 \mathrm{KHz}$.

Axial oblique DW imaging (oblique to the corpus) of the pelvis was performed using the single-shot echo-planar technique with fat suppression (TR/TE $5000 \mathrm{~ms} / 67.6 \mathrm{~ms}$, matrix $128 \times 128$, field of view $35 \times 35 \mathrm{~cm}$, number of acquired signals 4 , section thickness $5 \mathrm{~mm}$, and $b$ values 0 and $1000 \mathrm{~s} / \mathrm{mm}^{2}$ ). The array spatial sensitivity encoding technique was used as the parallel imaging technique during DW image scanning. The ADC map of each DW image was produced with a GE Advantage Windows (AW) 4.4 Workstation.

\section{Imaging analysis}

All MR sequences were randomized in order and viewed by two radiologists with 10 and 8 years of experience in gynecologic radiology, who were blinded to the histopathological findings and patients' names, but were aware that the patients had been diagnosed with endometrial cancer. Disagreements were resolved by consensus. For visual inspection, the readers evaluated the standard anatomic sequences (T1-and T2-weighted imaging) as well as 
the DW images for the depth of myometrial invasion, which was scored as 'superficial' if the tumor invaded up to $50 \%$ of the myometrial thickness and 'deep' if the tumor extended beyond $50 \%$ of the myometrium thickness. Tumor maximal diameter (as tumor size) [20-22] was calculated on multiple sequences, and the largest value was recorded. Quantitative analysis of DW images was performed using ADC maps which were generated on the scanner console using the $b=1000 \mathrm{~s} / \mathrm{mm}^{2}$ and $b=0 \mathrm{~s} /$ $\mathrm{mm}^{2}$ images. Regions of interest (ROIs) were applied to tumors and peritumoral zones. The peritumoral zone was defined as a 5 -mm-thick zone surrounding and adjacent to the cancerous endometrium [23]. The radiologists reviewed the T2 and DW images and determined the location of the deepest invasion of the tumor. An elliptical ROI (mean area, $20 \mathrm{~mm}^{2}$ ) was then drawn along the deepest invasion margin of the lesion for measuring the ADC of the peritumoral zone. The pictorial illustration of ROI placement on peritumoral zone is depicted in Fig. 1. For measuring the ADC of the tumor, ROIs were applied to the tumor that contained the largest endometrial cancerous area, avoiding artifacts from the neoplastic/non-neoplastic interface and visible lesions or vascular structures in the myometrium. The ROI setting was on the cross-section of the T2-weighted image obtained via echo planar imaging $\left(b=0 \mathrm{~s} / \mathrm{mm}^{2}\right)$, and it was manually copied to the corresponding ADC map, whereupon ADC values were automatically calculated (Fig. 2). For quality control, the placements of ROIs were determined by the two radiologists. Disagreements were resolved by consensus. The measurement was repeated three times and the interval between the measurements was 1 week. The final data recorded was $\mathrm{ADCm}$ (mean $\mathrm{ADC}$ ) value averaged from the three measurements by the two radiologists.

\section{Histopathological analysis}

All endometrial cancer patients underwent a total abdominal hysterectomy and bilateral salpingo-oophorectomy, including 20 patients who underwent pelvic lymphadenectomy simultaneously. After resection, the uterus was cut into $5 \mathrm{~mm}$-thick axial sections for evaluation of myometrial invasion depth, which was performed the same way as the MRI interpretation. A pathologist with 15 years of experience in gynecologic disease who was blinded to the imaging results assessed FIGO stage, histological type, tumor grade (G1, well differentiated; G2, moderately differentiated; and G3, poorly differentiated), and depth of myometrial invasion (superficial myometrial invasion, the tumor invades $<50 \%$ of the myometrium; deep myometrial invasion, the tumor invades $\geq 50 \%$ of the myometrium).

\section{Statistical analysis}

The ADCm values of the superficial and deep myometrial invasion of the tumor or peritumoral zone were compared using the independent sample $t$-test; two-tailed $p$ values of $<0.05$ were considered statistically significant. The cutoff $\mathrm{ADCm}$ value of the peritumoral zone in endometrial cancer was obtained by drawing a receiver operating characteristic (ROC) curve. In order to maximize both of sensitivity and specificity, we applied the Youden's index (Youden's index $=$ Sensitivity + Specificity-1). We chose the point closest to the upper left corner of the curve as a cutoff, where the Youden's index was maximal. The cutoff $\mathrm{ADCm}$ value and visual inspection were used as the diagnostic indexes to evaluate deep myometrial invasion. The sensitivity, specificity, positive predictive value (PPV), and negative predictive value (NPV) of the ADCm cutoff and visual inspection were calculated and represented with $95 \%$ confidence intervals. All statistical analyses were performed by IBM SPSS

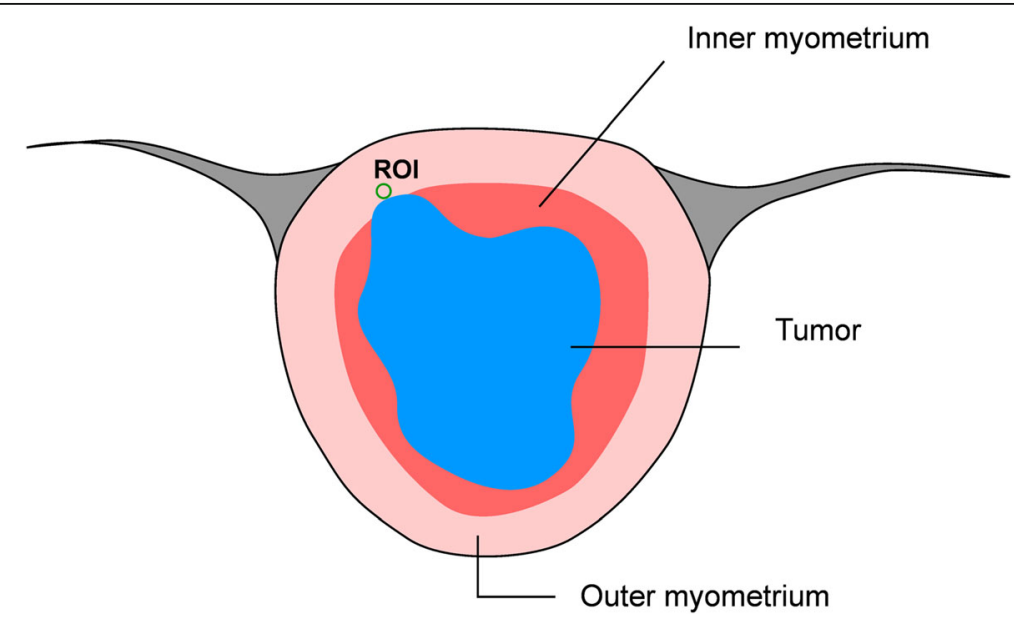

Fig. 1 The pictorial illustration of ROI placement on peritumoral zone of endometrial cancer. The tumor area is in blue, and the inner \& outer myometrium is in red\&pink. An elliptical region of interest (ROI) was drawn along the margin of deepest invasion of the tumor (i.e., peritumoral zone) 

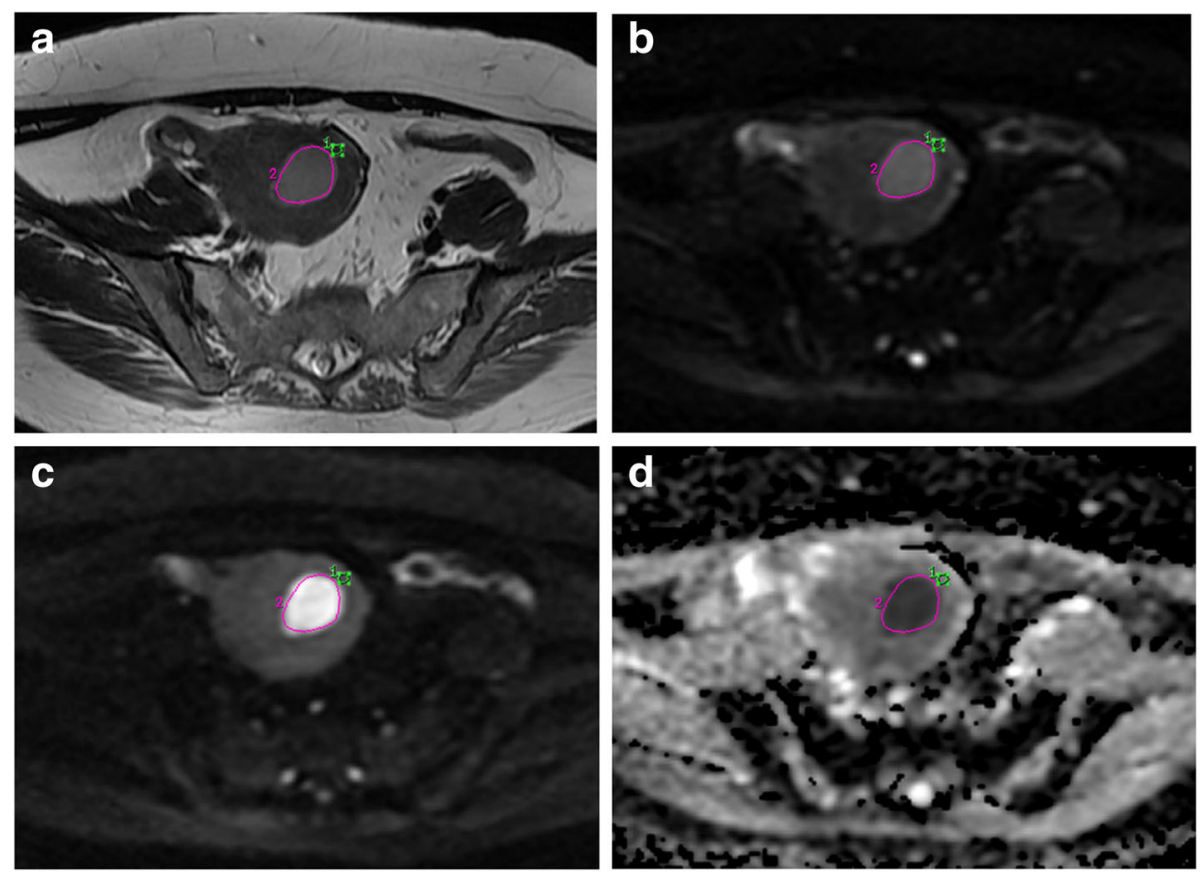

Fig. 2 Endometrial adenocarcinoma with superficial myometrial invasion in a 43-year-old woman. An elliptical region of interest (ROI) was placed on peritumoral zone ( $\mathrm{ROI}_{1}$ with green color), which was assessed subjectively on a cross-section of the T2-weighted image obtained by echo planar imaging $\left(b=0 \mathrm{~s} / \mathrm{mm}^{2}\right)$. In addition, a freehand $\mathrm{ROI}\left(\mathrm{ROI}_{2}\right.$ with pink color) was placed on the tumor which contained the largest endometrial cancerous area, avoiding artifacts from the neoplastic/non- neoplastic interface and visible lesions or vascular structures in the myometrium. a T2-weighted image; $\mathbf{b}$ diffusion-weighted magnetic resonance image $\left(b=0 \mathrm{~s} / \mathrm{mm}^{2}\right)$; c diffusion-weighted magnetic resonance image $\left(b=1000 \mathrm{~s} / \mathrm{mm}^{2}\right)$; $\mathbf{d}$ apparent diffusion coefficient map

statistical software, version 19.0. The ROC curve was drawn using Stata/SE 12.0 for windows. The pictorial illustration of the ROI placement was drawn by FREEHAND, version 11.0.2.

\section{Results}

\section{Histopathological findings}

The intervals between MRI examination and surgery were 0-21 days (mean 4 days). Of the 58 patients with endometrial cancer, postoperative histological assessment revealed endometrioid adenocarcinoma in 43, adenosquamous carcinoma in 11, mixed endometrioid/serous papillary carcinoma in 2, and mixed endometrioid/mucinous papillary carcinoma in 2. The tumor was confined to the endometrium or involved the inner half of the myometrium (superficial myometrial invasion) in 41 cases, and involved the outer half of the myometrium (deep myometrial invasion) in the remaining 17 cases. The relevant histopathological findings are shown in Table 1.

\section{Quantitative analysis}

Of the 58 endometrial cancers, the mean tumor size was $3.9 \pm 1.9 \mathrm{~cm}$. The ADCm values of tumor and the peritumoral zone were $(0.83 \pm 0.11) \times 10^{-3} \mathrm{~mm}^{2} / \mathrm{sec}$ and $(1.06 \pm$ $0.22) \times 10^{-3} \mathrm{~mm}^{2} / \mathrm{sec}$, respectively. There was no significant difference between the ADCm values of tumor in the superficial and deep myometrial invasion groups (superficial invasion, $0.84 \times 10^{-3} \mathrm{~mm}^{2} / \mathrm{sec}$ and deep invasion, $\left.0.82 \times 10^{-3} \mathrm{~mm}^{2} / \mathrm{sec} ; p>0.05\right)$. The $\mathrm{ADCm}$ value at the peritumoral zone of the deep myometrial invasion and that of the superficial myometrial invasion were $1.23 \times 10^{-}$ ${ }^{3} \mathrm{~mm}^{2} / \mathrm{sec}$ and $0.99 \times 10^{-3} \mathrm{~mm}^{2} / \mathrm{sec}$, respectively, which was a significant difference $(p=0.005)$ (Table 2 and Fig. 3).

Table 1 Patients' surgical and pathological findings

\begin{tabular}{lll}
\hline & Variable & Data \\
\hline Myometrial invasion & superficial & 41 \\
& deep & 17 \\
& endometrioid & 43 \\
Histological type & adenosquamous & 11 \\
& mixed endometrioid/mucinous papillary & 2 \\
Histological grade & 1 & 2 \\
& 2 & 13 \\
& 3 & 36
\end{tabular}

FIGO International federation of gynecology and obstetrics 
Table 2 Apparent diffusion coefficient values for different depth of myometrial invasion $\left(\times 10^{-3} \mathrm{~mm}^{2} / \mathrm{sec}\right)$

\begin{tabular}{llllll}
\hline & \multicolumn{2}{l}{ Peritumoral zone } & & \multicolumn{2}{l}{ Tumor } \\
\cline { 2 - 3 } & ADC & & $\mathrm{ADC}$ & $\mathrm{p}$ \\
\hline Superficial & $0.99 \pm 0.15$ & $0.005^{*}$ & & $0.84 \pm 0.10$ & $>0.05$ \\
Deep & $1.23 \pm 0.27$ & & & $0.82 \pm 0.14$ & \\
\hline
\end{tabular}

$A D C$ Apparent diffusion coefficient

${ }^{*} p<0.05$ was considered a statistically significant difference

\section{Diagnostic performance of the ADC cutoff value of the peritumoral zone and the visual inspection}

The diagnostic performances of the two methods for assessing deep myometrial involvement are summarized in Table 3. The ADC cutoff value of the peritumoral zone for assessing deep myometrial invasion was $1.17 \times 10^{-3} \mathrm{~mm}^{2} / \mathrm{sec}$. An additional figure file shows this in more detail [see Additional file 1]. For assessing deep myometrial invasion of endometrial cancer, the specificity for the ADCm cutoff value of the peritumoral zone (0.93) was higher than for visual inspection (0.80), as were the PPVs (ADCm, 0.77; vs. visual inspection, 0.60). The areas under the ROC curve $(\mathrm{Az})$ were 0.76 for both methods, but the diagnostic accuracy for the ADCm cutoff value (83\%) was higher than for visual inspection. The ROC curves are depicted in Fig. 4.

\section{Discussion}

Our results suggested that the ADCm of a tumor could not differentiate deep myometrial invasion from superficial myometrial invasion in endometrial cancer, which is concordant with previous researches [13, 14]. However, the ADCm of the peritumoral zone in the deep myometrial invasion group differed significantly from that of the superficial myometrial invasion group, and was therefore potentially useful for ruling out deep myometrial invasion. Moreover, it was more accurate than visual inspection for assessing deep myometrial invasion, and so could be used as a quantitative MRI tool for helping assess deep myometrial invasion of endometrial cancer.

DW imaging is a functional technique that provides information about water mobility, tissue cellularity, and the integrity of the cellular membrane. In biological tissues, water mobility, i.e., Brownian motion, is restricted via interaction with cell membranes and macromolecules at a microscopic level. In addition to providing essential qualitative information regarding the diffusivity of water molecules in a given tissue, DW imaging enables quantitative information to be obtained with the use of ADC maps [24]. Calculating the ADC can provide quantitative analysis of Brownian motion. The higher the signal of a region in a DW image, the lower the ADC values are, indicating thicker tissues with more densely populated cells [25].

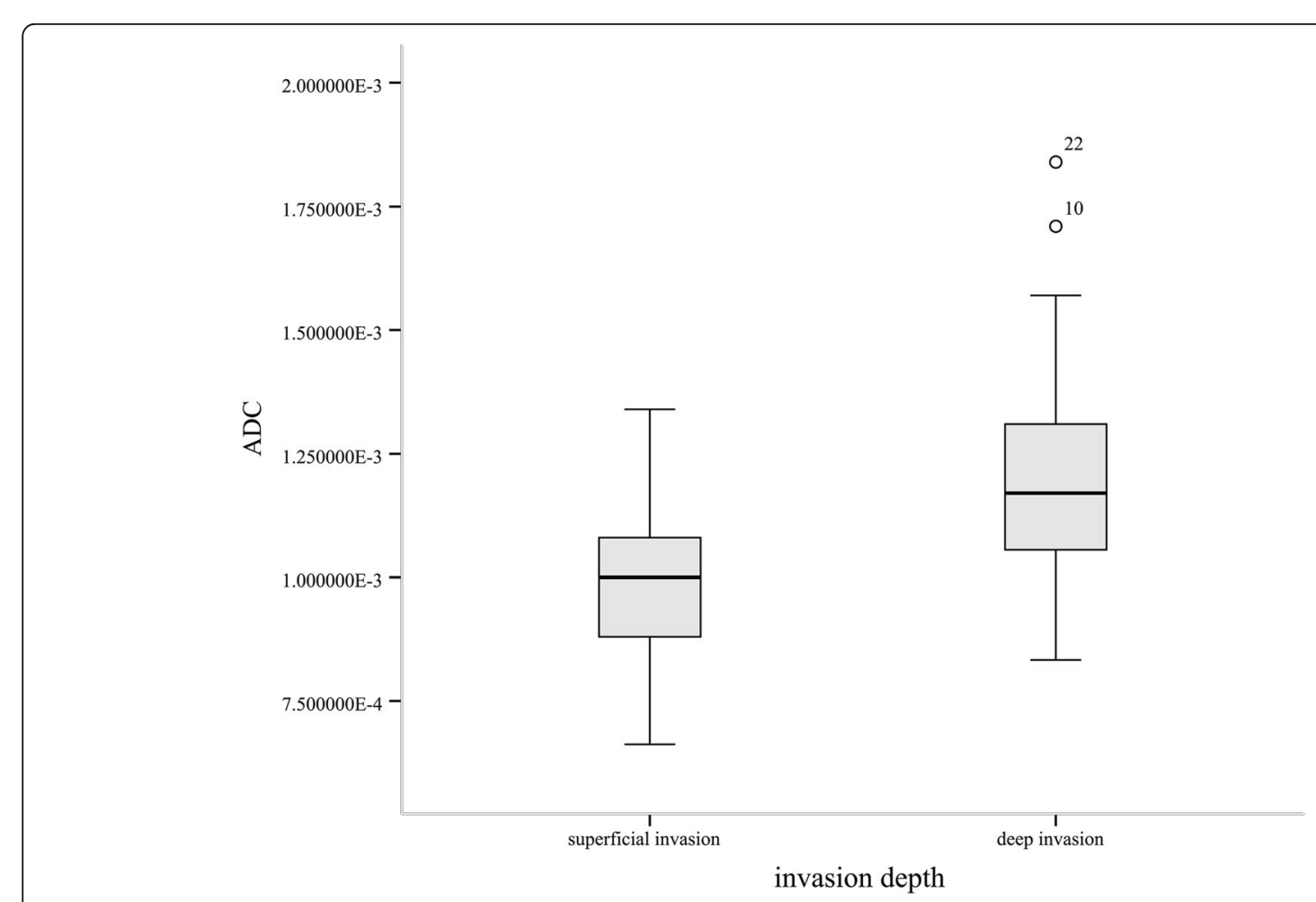

Fig. $\mathbf{3}$ It is a box and whisker plot which is the correlation between ADC value of peritumoral zone and histological depth of invasion 
Table 3 Diagnostic performance of deep myometrial invasion assessment by ADC cutoff value and visual inspection of peritumoral zone

\begin{tabular}{|c|c|c|c|c|c|c|c|c|c|c|}
\hline \multirow[t]{2}{*}{ Method } & \multicolumn{4}{|c|}{ Findings } & \multirow[t]{2}{*}{ Acc } & \multirow[t]{2}{*}{$\mathrm{Az}$} & \multirow{2}{*}{$\begin{array}{l}\text { Sensitivity } \\
(95 \% \mathrm{Cl})\end{array}$} & \multirow{2}{*}{$\begin{array}{l}\text { Specificity } \\
(95 \% \mathrm{Cl})\end{array}$} & \multirow{2}{*}{$\begin{array}{l}\text { NPV }(95 \% \\
\text { Cl) }\end{array}$} & \multirow{2}{*}{$\begin{array}{l}\text { PPV (95\% } \\
\text { CI) }\end{array}$} \\
\hline & $\mathrm{TP}$ & FP & $\mathrm{FN}$ & $\overline{\mathrm{TN}}$ & & & & & & \\
\hline \multirow[t]{2}{*}{ ADC cutoff } & 10 & 3 & 7 & 38 & 0.83 & 0.76 & 0.59 & 0.93 & 0.84 & 0.77 \\
\hline & & & & & & & $(0.33-0.81)$ & (0.79-0.98) & $(0.70-0.93)$ & $(0.46-0.94)$ \\
\hline \multirow[t]{2}{*}{ Visual inspection } & 12 & 8 & 5 & 33 & 0.78 & 0.76 & 0.71 & 0.80 & 0.87 & 0.60 \\
\hline & & & & & & & $(0.44-0.89)$ & $(0.65-0.91)$ & $(0.71-0.95)$ & $(0.36-0.80)$ \\
\hline
\end{tabular}

Data are means and numbers in parentheses are $95 \%$ confidence intervals

ADC Apparent diffusion coefficient, Az Area under the receiver operating characteristic curve, Acc Accuracy, NPV Negative predictive value, PPV Positive predictive value, $\mathrm{Cl}$ Confidence interval, TP True-positive, FP False-positive, FN False-negative, TN True-negative

In this study, the peritumoral zone was defined as a 5-mm-thick zone surrounding and adjacent to the cancerous endometrium. In patients with normal endometrium, it is the junctional zone of the uterus. Three distinct layers can be visualized via T2-weighted MRI in a normal uterus: a high signal intensity layer corresponding to the endometrial stripe, an inner low signal intensity layer that is adjacent to the basal endometrium (the junctional zone or subendometrial layer), and an outer medium signal intensity subserosal zone or outer myometrium [26]. The junctional zone has increased nuclear area, decreased extracellular matrix, and lower water content in comparison with the outer myometrium. In addition, junctional zone myocytes are thought to express different extracellular matrix components [27, 28]. These features not only shorten the T2 but also restrict diffusion, which gives rise to a low signal zone on the ADC map and the lowest ADC value of this region in the normal uterus [18]. Previous studies showed that in the normal uterus, the ADC value of the junctional zone was the lowest among the three layers and that of the outer myometrium was the highest $[18,19]$. In deep myometrium infiltration of endometrial cancer, the tumor invades $\geq 50 \%$ of the myometrium and thus appears as a complete disruption of the junctional zone. When this happens, the peritumoral zone actually includes a majority of the outer myometrium and a small amount of cancerous tissue, which is indicated by a higher signal zone on T2-weighted imaging and ADC maps in comparison with the normal junctional zone. In contrast, in superficial myometrial invasion $(<50 \%$ of myometrium invasion depth), the peritumoral zone consists of partial junctional zone, partial outer myometrium, and a small amount of cancerous tissue which exhibit a lower signal in comparison with deep myometrial invasion on T2-weighted imaging and ADC maps. Accordingly, there should be a restricted diffusion difference between deep and superficial myometrial invasion. This was confirmed by our result showing that the ADCm of peritumoral zone of deep myometrial invasion $\left(1.23 \times 10^{-3} \mathrm{~mm}^{2} / \mathrm{sec}\right)$ was significantly greater than that of superficial myometrial invasion $\left(0.99 \times 10^{-3} \mathrm{~mm}^{2} /\right.$ sec) $(p=0.005)$. Thus, the ADCm value of the peritumoral zone may provide useful information for differentiating deep myometrial invasion from superficial myometrial invasion in endometrial cancer.

In the current study, in endometrial cancer patients, the $\mathrm{ADCm}$ values of the tumor exhibiting deep myometrial invasion and superficial myometrial invasion did not differ significantly. This finding is concordant with results previously reported by Lin et al. [14] and Rechichi et al. [13]. A possible explanation for this finding is that cellular density

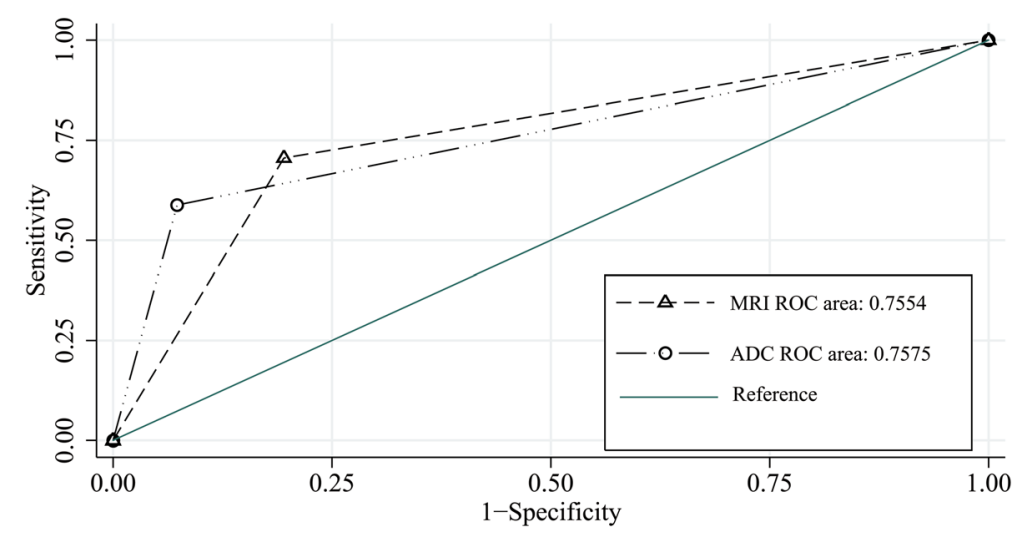

Fig. $4 \mathrm{Az}$ values of the two methods obtained from $\mathrm{ROC}$ 
and medium interstice are the main factors affecting Brownian motion. A tissue with high cellular density and medium interstice, such as neoplastic tissue, corresponds to high signal in DW imaging. Conversely, tissue with lower signal in DW imaging (i.e., normal issue) corresponds to a region with a higher ADC value [24]. Notably however, other important features of tumor cells such as nuclear atypia cannot be assessed by DW imaging [29]. That is, the $A D C$ value alone is not sufficient for ascertaining the invasiveness of a tumor.

The current study had some limitations. One was the small size of the deep myometrial invasion group. The deep infiltration group included 17 patients $(29.3 \%$ of the entire study group), which might have biased the sensitivity, specificity, PPV, and NPV of the two assessment methods such that they did not reach statistical significance. Moreover, the study lacked objective assessments to determine the location of the deepest myometrial invasion where an ROI should be set.

\section{Conclusion}

The ADC value obtained at the peritumoral zone can predict deep myometrial invasion of endometrial cancer. This value could therefore enhance confidence in the preoperative evaluation of endometrial cancer, and be useful when tailoring the surgical approach.

\section{Additional file}

Additional file 1: A ROC curve of the ADCm value of the periturmoral zone in endometrial cancer. The cutoff ADCm value of the peritumoral zone was obtained from the curve. We chose the point closest to the upper left corner of the curve as a cutoff, where the Youden's index (Youden's index = Sensitivity+Specificity-1) was maximal. (TIF $1474 \mathrm{~kb}$ )

\section{Abbreviations}

ADC: Apparent diffusion coefficient; ADCm: Mean apparent diffusion coefficient; DW: Diffusion-weighted; FIGO: Federation of Gynecology and Obstetrics; MRI: Magnetic resonance imaging; NPV: Negative predictive value; PPV: Positive predictive value; ROC: Receiver operating characteristic; ROI: Region of interest; TE: Echo time; TR: Repetition time

\section{Acknowledgements}

We would like to thank Editage (www.editage.cn) for English language editing, and Mr. Tao Song for the pictorial illustration.

\section{Funding}

Not applicable.

\section{Availability of data and materials}

The datasets generated and/or analyzed during the current study are not publicly available due [the protecting provision about the patients' privacy included in informed consent] but are available from the corresponding author on reasonable request.

\section{Authors' contributions}

$L D, L B$ and RY made substantial contributions to conception and design, acquisition of data, analysis and interpretation of data; NY, X-yD and Q-pW were involved in drafting the manuscript. Q-XY and $Y-m G$ gave final approval of the version to be published. Q-XY agreed to be accountable for all aspects of the work in ensuring that questions related to the accuracy or integrity of any part of the work are appropriately investigated and resolved. All authors read and approved the final manuscript.

Ethics approval and consent to participate

The work was carried out at the First Affiliated Hospital of Xi'an Jiaotong University. The ethics committee of the First Affiliated Hospital of Xi'an Jiaotong University approved this prospective study, and all study participants provided informed consent.

\section{Consent for publication}

The current study contained one patient's MR images. This patient had signed the consent form for agreement of publishing.

\section{Competing interests}

The authors declare that they have no competing interests.

\section{Publisher's Note}

Springer Nature remains neutral with regard to jurisdictional claims in published maps and institutional affiliations.

\section{Author details}

${ }^{1}$ Department of Radiology, the First Affiliated Hospital, Xi'an Jiaotong University Xi'an, \#277, Yanta West Road, Xi'an 710061, Shaanxi, China. ${ }^{2}$ Department of Radiology, the Northwest Women and Children Hospital, \#1616, Yanxiang Road, Xi'an 710054, Shaanxi, China. ${ }^{3}$ Department of Nuclear Medicine, the First Affiliated Hospital, Xi'an Jiaotong University Xi'an, \#277, Yanta West Road, Xi'an 710061, Shaanxi, China. ${ }^{4}$ Department of Radiology, The Affiliated Hospital of Shaanxi University of traditional Chinese Medicine, \#2. Wei Yang West Road, Xian Yang 712000, Shaanxi, China.

Received: 7 July 2017 Accepted: 22 June 2018

Published online: 03 July 2018

\section{References}

1. Siegel R, Ma J, Zou Z, Jemal A. Cancer statistics, 2014. CA Cancer J Clin. 2014;64(1):9-29.

2. Burke WM, Orr J, Leitao M, Salom E, Gehrig P, Olawaiye AB, Brewer M, Boruta D, Villella J, Herzog T, et al. Endometrial cancer: a review and current management strategies: part I. Gynecol Oncol. 2014;134(2):385-92.

3. Rockall AG, Meroni R, Sohaib SA, Reynolds K, Alexander-Sefre F, Shepherd $\mathrm{JH}$, Jacobs I, Reznek RH. Evaluation of endometrial carcinoma on magnetic resonance imaging. Int J Gynecol Cancer. 2007;17(1):188-96.

4. Larson DM, Connor GP, Broste SK, Krawisz BR, Johnson KK. Prognostic significance of gross myometrial invasion with endometrial cancer. Obstet Gynecol. 1996;88(3):394-8.

5. Berman ML, Ballon SC, Lagasse LD, Watring WG. Prognosis and treatment of endometrial cancer. Am J Obstet Gynecol. 1980;136(5):679-88.

6. Morice P, Leary A, Creutzberg C, Abu-Rustum N, Darai E. Endometrial cancer Lancet. 2015;

7. Hardesty. Use of preoperative MR imaging in the Management of Endometrial Carcinoma Cost Analysis. Radiology. 2000;215(1)

8. Thoeny HC, Forstner R, De Keyzer F. Genitourinary applications of diffusionweighted MR imaging in the pelvis. Radiology. 2012;263(2):326-42.

9. Gallego JC, Porta A, Pardo MC, Fernandez C. Evaluation of myometrial invasion in endometrial cancer: comparison of diffusion-weighted magnetic resonance and intraoperative frozen sections. Abdom Imaging. 2014;39(5): 1021-6.

10. Das SK, Niu XK, Wang JL, Zeng LC, Wang WX, Bhetuwal A, Yang HF. Usefulness of DWI in preoperative assessment of deep myometrial invasion in patients with endometrial carcinoma: a systematic review and metaanalysis. Cancer Imaging. 2014;14(1):32.

11. Deng L, Wang Q-p, Chen X, Duan X-y, Wang W, Guo Y-m. The combination of diffusion- and T2-weighted imaging in predicting deep myometrial invasion of endometrial Cancer: a systematic review and meta-analysis. J Comput Assist Tomogr. 2015;39(5):661-73.

12. Fujii S, Matsusue E, Kigawa J, Sato S, Kanasaki Y, Nakanishi J, Sugihara S, Kaminou T, Terakawa N, Ogawa T. Diagnostic accuracy of the apparent diffusion coefficient in differentiating benign from malignant uterine endometrial cavity lesions: initial results. Eur Radiol. 2008;18(2):384-9.

13. Rechichi G, Galimberti S, Signorelli M, Franzesi CT, Perego P, Valsecchi MG, Sironi S. Endometrial cancer: correlation of apparent diffusion coefficient 
with tumor grade, depth of myometrial invasion, and presence of lymph node metastases. AJR Am J Roentgenol. 2011;197(1):256-62.

14. Lin G, Ng KK, Chang CJ, Wang JJ, Ho KC, Yen TC, Wu TI, Wang CC, Chen YR, Huang YT, et al. Myometrial invasion in endometrial cancer: diagnostic accuracy of diffusion-weighted 3.0-T MR imaging-initial experience. Radiology. 2009;250(3):784-92.

15. Pecorelli S. Revised FIGO staging for carcinoma of the vulva, cervix, and endometrium. Int J Gynaecol Obstet. 2009;105(2):103-4.

16. Beddy P, O'Neill AC, Yamamoto AK, Addley HC, Reinhold C, Sala E. FIGO staging system for endometrial cancer: added benefits of MR imaging. Radiographics. 2012;32(1):241-54.

17. Sala E, Rockall A, Kubik-Huch RA. Advances in magnetic resonance imaging of endometrial cancer. Eur Radiol. 2011;21(3):468-73.

18. Kuang F, Ren J, Huan Y, Chen Z, Zhong Q. Apparent diffusion coefficients of normal uterus in premenopausal women with 3.0-T magnetic resonance imaging. J Comput Assist Tomogr. 2012;36(1):54-9.

19. Kilickesmez O, Bayramoglu S, Inci E, Cimilli T, Kayhan A. Quantitative diffusion-weighted magnetic resonance imaging of normal and diseased uterine zones. Acta Radiol (Stockholm, Sweden: 1987). 2009;50(3):340-7.

20. Bourgioti C, Chatoupis K, Tzavara C, Antoniou A, Rodolakis A, Moulopoulos LA. Predictive ability of maximal tumor diameter on MRI for high-risk endometrial cancer. Abdom Radiol (NY). 2016:41(12):2484-95.

21. Yang Y, Zhao L, Wang Z, Tang J, Geng J, Hong N, Wang J, Wei L. Clinical value of transvaginal ultrasound, MRI and hysteroscopy in the assessment of endometrial cancer lesion size. Zhonghua fu chan ke za zhi. 2016;51(1):36-9.

22. Berretta R, Patrelli TS, Migliavacca C, Rolla M, Franchi L, Monica M, Modena $A B$, Gizzo $S$. Assessment of tumor size as a useful marker for the surgical staging of endometrial cancer. Oncol Rep. 2014;31(5):2407-12.

23. McLaughlin RL, Newitt DC, Wilmes LJ, Jones EF, Wisner DJ, Kornak J, Proctor E, Joe BN, Hylton NM. High resolution in vivo characterization of apparent diffusion coefficient at the tumor-stromal boundary of breast carcinomas: a pilot study to assess treatment response using proximity-dependent diffusion-weighted imaging. J Magn Reson Imaging. 2014;39(5):1308-13.

24. Colagrande S, Pallotta S, Vanzulli A, Napolitano M, Villari N. The diffusion parameter in magnetic resonance: physics, techniques, and semeiotics. La Radiologia Medica. 2005;109(1-2):1-16.

25. Manenti G, Di Roma M, Mancino S, Bartolucci D, Palmieri G, Mastrangeli R, Miano R, Squillaci E, Simonetti G. Malignant renal neoplasms: correlation between $A D C$ values and cellularity in diffusion weighted magnetic resonance imaging at 3 T. La Radiologia Medica. 2008;113(2):199-213.

26. Fusi L, Cloke B, Brosens JJ. The uterine junctional zone. Best Pract Res Clin Obstet Gynaecol. 2006;20(4):479-91.

27. McCarthy S, Scott G, Majumdar S, Shapiro B, Thompson S, Lange R, Gore J. Uterine junctional zone: MR study of water content and relaxation properties. Radiology. 1989;171(1):241-3.

28. Scoutt LM, Flynn SD, Luthringer DJ, McCauley TR, McCarthy SM. Junctional zone of the uterus: correlation of MR imaging and histologic examination of hysterectomy specimens. Radiology. 1991;179(2):403-7.

29. Whittaker CS, Coady A, Culver L, Rustin G, Padwick M, Padhani AR. Diffusionweighted MR imaging of female pelvic tumors: a pictorial review. Radiographics. 2009;29(3):759-74. discussion 774-758

\section{Ready to submit your research? Choose BMC and benefit from:}

- fast, convenient online submission

- thorough peer review by experienced researchers in your field

- rapid publication on acceptance

- support for research data, including large and complex data types

- gold Open Access which fosters wider collaboration and increased citations

- maximum visibility for your research: over $100 \mathrm{M}$ website views per year

At BMC, research is always in progress.

Learn more biomedcentral.com/submissions 